\title{
Placental pathology in different degrees of pregnancy induced hypertension (PIH)
}

\author{
S. Rajyalakshmi ${ }^{1}$, Vani B $S^{2}$, Jijiya Bai $P^{3}$ \\ ${ }^{1}$ Dr S. Rajyalakshmi, Associate Professor, ${ }^{2}$ Dr Vani BS, Assistant Professor, ${ }^{3}$ Dr Jijiya Bai P., Professor and Head, all authors \\ are affiliated to Department of Pathology, Mallareddy Medical College for Women, Suraram, Hyderabad, Telangana, India.
}

Corresponding Author: Dr Vani BS, Assistant Professor, Department of Pathology, Mallareddy Medical College for Women, Suraram, Hyderabad, Telangana, India. E-mail: vanibs76@rediffmail.com

\begin{abstract}
Introduction: Pregnancy induced hypertension $(\mathrm{PIH})$, a well-known medical complication of pregnancy is potentially morbid for the foeto-maternal health. It is also responsible for perinatal morbidity and mortality due to its effects on the growing foetus. Objectives: The present study was undertaken to analyse the histomorphological and gross features of placenta in pregnancy induced hypertension in relation to severity of hypertension, age of the patient, parity and gestational age. Materials and Methods: The current study was done at Gandhi Hospital, Secunderabad, India on antenatal cases of PIH of varying degrees. The placentas of these patients following delivery were studied and statistic significance between different histologic findings and severity of PIH calculated. Results: Incidence of PIH was highest in women above 26 years of age $(42 \%)$ and was found to be almost equal in both multiparous (52\%) and primiparous (48\%) women. Foetal outcome was worst in severe PIH (IUGR of $28.8 \%$ and IUD of $17.85 \%)$. The gross abnormalities noted, retroplacental hematomas (42\%) and small sized placentae (24\%) were more often seen in severe PIH. The consistent histological changes observed include fibrinoid necrosis (86\%), thickened basement membrane (74\%), increased syncitial knots (72\%), stromal fibrosis (54\%), cytotrophoblastic proliferation $(46 \%)$, intervillous haemorrhage (40\%), and calcification (34\%). Conclusion: The pathological changes in the placenta seen in PIH patients are almost equally prevalent irrespective of the parity. Cases with severe PIH displayed features of placental under perfusion more frequently than with mild to moderate PIH. Hence watchful individualized management of PIH helps reduce the incidence of complications and morbidity.
\end{abstract}

Keywords: PIH, Placental pathology, Maternal under perfusion, Foetal vasculopathy, Villous abnormalities, vascular abnormalities

\section{Introduction}

The wellbeing of the mother and baby is of utmost importance to the economy of a country. The antenatal examination is the most appropriate mode of prevention of disorders associated with pregnancy. Hypertension is one of the common disorders encountered in pregnancy which can cause significant morbidity and mortality. It is proposed that maternal and placental factors contribute to hypertension during pregnancy and to pre-eclampsia, a syndrome of pregnancy induced hypertension that develops after 20 weeks of gestation associated with proteinuria [1]. Pre-eclampsia with seizures is called ecclampsia. Pathologic processes interfering with placental function may result in abnormalities of fetal growth or development, malformation, or stillbirth, and there is increasing recognition that some long-term (especially neurologic) disabilities can be traced to injury occurring before birth [2].

Manuscript received: $5^{\text {th }}$ October 2019

Reviewed: $15^{\text {th }}$ November 2019

Author Corrected: $20^{\text {th }}$ November 2019

Accepted for Publication: $26^{\text {th }}$ November 2019
Hence perinatal mortality and morbidity correlates with the extent of placental lesions and the context in which these lesions impact the health of the mother, the fetus or both. Ezeigwe et al also have proposed that significant pathology in placenta is inversely proportional to foetal birth weight contributing to perinatal morbidity [3]. PIH has been documented to be associated with low birth weight and stillbirth in various studies [4].

Pathologic processes affecting the vessels and spaces in and around the placenta may cause both fetal and maternal injury depending on their size, location, and extent. Measurement of size and evaluation of extent is important in assessing the significance of individual lesions. The aim of this study was to determine the placental changes in varying degrees of pregnancy induced hypertension and identify the epidemiology of foetal and maternal morbidity so as to determine timely preventive and therapeutic interventions in the risk group.

Pathology Update: Tropical Journal of Pathology \& Microbiology Available online at: www.medresearch.in 988 | P a g e 


\section{Materials and Methods}

Study design: A prospective study of 50 cases of pregnancy induced hypertension (PIH) in Gandhi Hospital was conducted in collaboration with Department of Gynecology and Obstetrics, Gandhi Hospital, Secunderabad. PIH cases were evaluated both clinically and by routine laboratory investigations. After delivery, placentas of pregnancy induced hypertension cases were received and examined for placental morphology in relation to varying degrees of PIH.

Definitions: According to NHS guidelines PIH is defined as a blood pressure- systolic $\geq 140 \mathrm{~mm}$ of $\mathrm{Hg}$ and/or diastolic $\geq 90 \mathrm{~mm}$ of $\mathrm{Hg}$. It is graded as i) Mild: $140 / 90$ to $149 / 99 \mathrm{~mm}$ of $\mathrm{Hg}$, ii) Moderate: 150/100 -159/109 mm of $\mathrm{Hg}$ and iii) Severe: $\geq 160 / 110 \mathrm{~mm}$ of $\mathrm{Hg}$.

Placental Pathology: The placentas received in the lab were examined in a fresh state. Examination of the membranes, umbilical cord, foetal surface and maternal surface was done to identify gross abnormalities; placentas were examined for the following parameters:

- Measurement of the diameter and thickness.

- Weight after fetal membranes, umbilical cord and large clots have been removed. Formalin fixation adds about $10 \%$ to placental weight.

- Anomalous shapes.

\section{Results}

Of the 50 cases of pregnancy induced hypertension analysed in the present study $6(12 \%)$ cases were of mild pregnancy induced hypertension, $16(32 \%)$ cases were of moderate pregnancy induced hypertension and $28(56 \%)$ cases were of severe pregnancy induced hypertension.

Age wise analysis of cases: Age of the patients varied from 16 years to 30 years. Incidence of pregnancy induced hypertension was found to be more above 26 years of age (Figure 1).

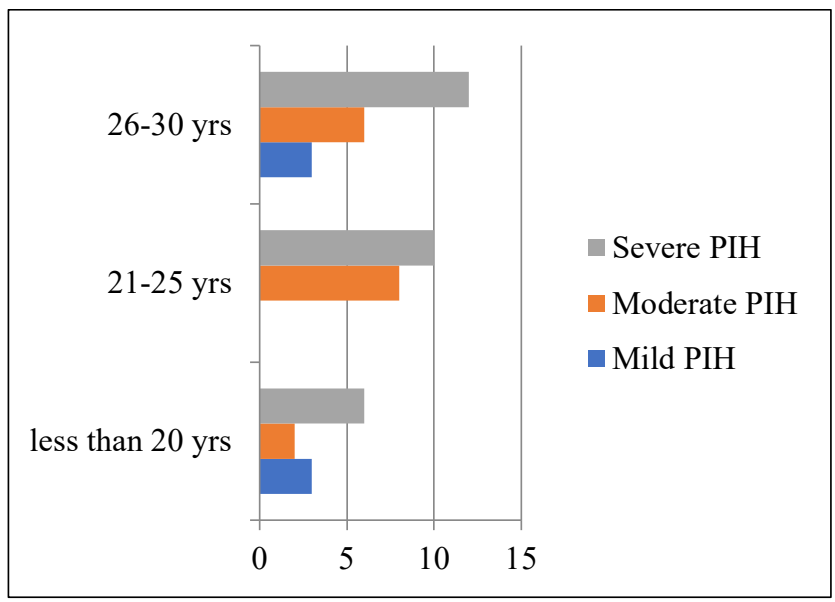

Fig-1: Age wise analysis of cases of pregnancy induced hypertension.

Parity: Incidence of pregnancy induced hypertension was found to be almost equal in both primiparous and multiparous women (Table 1).

Pathology Update: Tropical Journal of Pathology \& Microbiology Available online at: www.medresearch.in 989 | P a g e 
Table-1: Parity in 50 cases of pregnancy induced hypertension.

\begin{tabular}{|c|c|c|c|c|c|}
\hline Parity & Mild PIH & Moderate PIH & Severe PIH & Total & Percentage \\
\hline Primipara & 3 & 10 & 11 & 24 & $48 \%$ \\
\hline Multipara & 3 & 6 & 17 & 26 & $52 \%$ \\
\hline
\end{tabular}

Foetal outcome: Mild pregnancy induced hypertension did not show any changes in foetus. A gradual rise in the number of I.U.G.R cases and IUDs wer seen from moderate to severe PIH (Table 2).

Table-2: Foetal Outcome in 50 cases of pregnancy induced hypertension.

\begin{tabular}{|l|c|c|c|}
\hline Foetal outcome & Mild PIH (6) & Moderate PIH (16) & Severe PIH (28) \\
\cline { 1 - 2 } Normal live births & 6 & 10 & 15 \\
\cline { 1 - 2 } IUGR & - & 4 & 8 \\
\cline { 1 - 1 } IUD & - & 2 & 5 \\
\hline
\end{tabular}

Gross abnormalities: The gross abnormalities found in the present study were retroplacental hematoma and small sized placenta. Retroplacental hematomas were more common than small sized placenta. Cases of mild pregnancy induced hypertension did not show any morphological changes in placenta. Morphological changes were found more frequently in severe than the moderate pregnancy induced hypertension cases (Table 3).

Table-3: Gross changes observed in placentas of pregnancy induced hypertension.

\begin{tabular}{|l|c|c|c|}
\hline Gross appearance & Mild PIH & Moderate PIH & Severe PIH \\
\hline Retroplacental haematomas & 0 & 5 & 16 \\
\hline Small placenta & 0 & 3 & 9 \\
\hline
\end{tabular}

One case of twin pregnancy was observed in severe pregnancy induced hypertension. In this case, the placenta was monoamniotic and monochorionic with double cord. Retroplacental haematomas were noted in this case. Babies were full term I.U.Ds.

Histomorphological changes (Figure 2): Analysis was done depending on the grading of hypertension. Most common histological findings in different degrees of PIH were:

Mild PIH - Increased syncitial knots in 4 (66.6\%) and thickened basement membrane in $3(50 \%)$ cases.

Moderate PIH-Thickened basement membrane in $14(87.5 \%)$ and areas of fibrinoid necrosis in $13(81.2 \%)$ cases.

Severe PIH- Fibrinoid Necrosis in 27 (96.4\%), syncitial knots in 23 (82.1\%) followed by thickened basement membrane in 20 $(71.5 \%)$ cases.

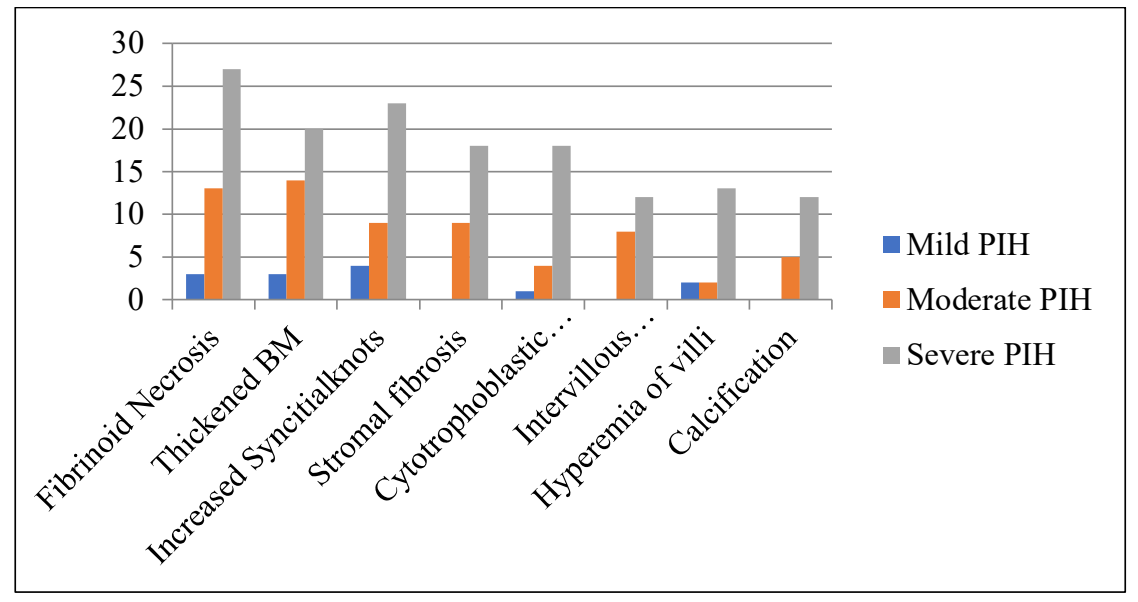

Fig-2: Distribution of histomorphological changes in placenta in varying degrees of PIH

Pathology Update: Tropical Journal of Pathology \& Microbiology Available online at: www.medresearch.in 990 | P a g e 
The severity and frequency of histomorphological changes increased as the severity of pregnancy induced hypertension progressed from mild through moderate to severe PIH. Neverthless, out of all the histological changes evaluated, the association of fibrinoid necrosis and cytotrophoblastic proliferation with the severity of PIH was found to be statistically significant ( $\mathrm{p}<$ 0.05). The other microscopic lesions were independent of the degree of PIH in the present study.

The following special stains were undertaken wherever necessary.

1. Periodic Acid Schiff stain-PAS positivity was seen in fibrinoid necrosis

2. Reticulin Stain -positivity was seen in the reticulin fibers in areas of fibrosis

3. Van Gieson Stain - was used for stromal fibrosis

\section{Discussion}

The placenta is considered to be a very complex organ with partially understood functions [1]. Placental changes in disorders of pregnancy is reflected in macroscopic and microscopic abnormalities. They also vary with the intensity of pathophysiologic mechanisms.

Macroscopic abnormalities: The morphological changes in the placenta have been attributed to both physiological and pathological conditions. Depending upon the incidence and severity of changes in large study groups, certain findings are considered pathological.

The predominant macroscopic abnormality found in this study was retroplacental hematomas. Retroplacental hematoma is a hematoma between the placental floor and myometrium. It represents the pathologic lesion responsible for clinical placental abruption. The pathogenesis is presumed to be due to a bleeding from a decidual artery followed by enlargement of the clot and dissection. Retroplacental hematoma is encountered three times more frequently in pre-eclampsia presumably due to the underlying decidual vasculopathy. It could also result from trauma. The significance of hematoma depends on the size of the clot [2]. H Fox has described that retroplacental hematomas are only associated with fetal deprivation if more than a third of the placental parenchyma is separated by the hematoma from the maternal uteroplacental vessels or if the bleeding occurs in a pregnancy already complicated by a hypertensive condition [5].

Large clots indent the placenta and separate it from its blood supply causing placental infarctions. Extensive recent hemorrhage causes fetal distress necessitating immediate delivery [2]. Large hematomas depriving $40 \%$ or more of the villous population of its blood supply are associated with high incidence of fetal hypoxia and death [6].

In 21 out of 50 cases studied (42\%) showing retroplacental hematomas, 5 cases were in moderate PIH and 16 were seen in severe $\mathrm{PIH}$, thus explaining the increased fetal morbidity in severe PIH. The incidence of retroplacental hematomas in other studies were $32.5 \%$ [6] and $40.7 \%$ [7]. In study conducted by Maimoona, none of the mild PIH cases had a hematoma; $10 \%$ of moderate and $36.4 \%$ of severe PIH cases had retroplacental hematoma [8].

The other gross abnormality found was the small-sized placentas

Two types of abnormal placentas are associated with preeclampsia. The first and most common is the small placenta with decidual vasculopathy, especially acute atherosis, the pathologic changes characteristic of maternal vascular under perfusion, and thin umbilical cord. Doppler velocimetry has identified growth-restricted fetuses with fetal vascular narrowing and increased vascular resistance in cases with small placentae [2]. Small placentae are seen in pre-eclampsia more often than in normal deliveries [5].

In the present study out of 50 cases of pregnancy induced hypertension small placenta was seen in 12 cases (24\%), out of which 3 cases were of moderate and 9 cases were of severe PIH. Bar et al have documented a statistically significant low values of placental diameter in hypertensive pregnancies compared to normal ones [9]. Karun Jain et al have shown that smaller babies had smaller placentas [10]. Maimoona and Rekha came across a significant negative correlation between placental thickness and PIH severity i.e. as the PIH severity increased, the placental thickness decreased [8].

Microscopic abnormalities: Many studies have established the different histologic findings in hypertensive disorders of pregnancy. The present study showed the following histologic features (Figure 3).

Pathology Update: Tropical Journal of Pathology \& Microbiology Available online at: www.medresearch.in 991 | P a g e 
A) Fibrinoid necrosis: Fibrin has immunohistochemical features of blood clot product and accumulates in maternal vascular under perfusion associated with decidual vasculopathy and the syndrome of preeclampsia. The fact that the intervillous space is involved would seem to implicate a maternal circulatory abnormality. Isolated reports have identified a group of associations including antiphospholipid antibody syndrome, polymyositis, long-chain L-3-hydroxyacyl-coA dehydrogenase deficiency, chronic intervillositis, and thrombophilias. Two separate reports, however, document involvement of only one of dichorionic twins, suggesting a role for the fetus. Whether the fibrinoid material that encases the terminal villi in these disorders reflects coagulation secondary to stasis of maternal blood in the intervillous space or aberrant secretion of matrix by villous trophoblast is unknown. Morphologically, wide strands and interlacing clumps of hard waxy pale tan or gray material accumulate as a thickened rind in the basal portion and ramify throughout the placenta. When most of the material is concentrated basally, the term MFI best applies, even though other parts of the placenta may also be involved. In MPF, the deposition of fibrinoid is diffuse without preferential basal concentration, although there may be patchy, sometimes extensive involvement of the maternal floor as part of the process. Microscopically, the villi are widely separated by pink amorphous matrix-type fibrinoid containing numerous mononuclear trophoblast cells. The syncytiotrophoblast and capillary endothelium disappear from entrapped villi, but the villous stroma and villous outlines persist. Villi entrapped in fibrinoid necrosis are isolated and non-functional. MFI and MPF represent the most severe end of a continuum. Redline and Patterson observed that perivillous fibrin deposits that entrapped more than $20 \%$ of villi in the central basal portion of the placenta (thought to be the primary region of gas and nutrient exchange) were significantly associated with an IUGR and low placental weight. Milder cases of perivillous fibrin deposition enveloping $5-20 \%$ of terminal villi showed a lesser degree of similar complications. Fibrin deposits at the placental margin or beneath the chorionic plate do not appear to have any adverse affect on the fetus (Figure 3) [2].

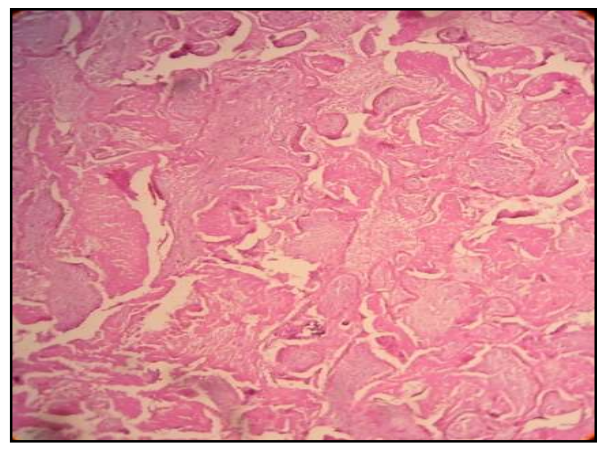

Fig-3: Fibrinoid necrosis of villi (H\&E 10X).

Although the exact mechanism is not understood, it is proposed to be due to degeneration from placental aging or hypoxic damage to trophoblasts [11]. It has also been attributed to an immunological reaction within villous tissue and to amyloid deposition as an ageing change [6].

In the present study, out of 6 cases of mild pregnancy induced hypertension, intervillous fibrinoid necrosis was seen in 3 cases (50\%) of mild, 13 cases $(81.2 \%)$ of moderate and 27 cases $(96.4 \%)$ of severe pregnancy induced hypertension. In the study by Mohan et al, fibrinoid necrosis was $28.9 \%$ of all high risk pregnancies [11]. According to Ananya and others fibrinoid necrosis was seen in more than $3 \%$ of villi in $77.5 \%$ of cases of preeclampsia and eclampsia [6]. Narsimha et al noted significant villous fibrinoid necrosis in $97.82 \%$ cases of preeclampsia-eclampsia syndromes [4].

B) Thickening of Basement Membrane (BM): Thickened basement membrane in more than 3\% of villous population is regarded as abnormal and is a common feature of toxemia. It occurs due to cytotrophoblast cell hyperplasia as the BM protein is deposited by these cells [7]. Kumar Abbas and Fausto in 2004 described the thickening of the trophoblastic basement membrane, striking fibrinoid necrosis, intramural lipid deposition (acute atherosis) and villous hypo-vascularity a characteristic finding in the walls of uterine vessels, in case of pregnancy induced hypertension [12].

In the present study, 3 cases (50\%) of mild, 14 cases $(87.5 \%)$ of moderate and $20(71.5 \%)$ cases of severe pregnancy induced hypertension showed thickening of basement membrane. Narsimha et al have demonstrated that basement membrane thickening progressed from $4 \%$ in mild preeclampsia to $20 \%$ in severe preeclampsia to $100 \%$ of eclampsia cases [7].Ananya and team found significant increasing association of trophoblast basement membrane thickening with grades of hypertension from mild preeclampsia through severe preeclampsia, eclampsia and pre-eclampsia superimposed on essential hypertension [6] 50\% and $55.2 \%$ of PIH cases showed BM thickening in a study by Bar et al and Mohan et al respectively $[9,11]$. 
C) Increased syncitial knots: Syncitial knots are focal clumps of syncitial nuclei that protrude into the intervillous space from the surface of the villi. Syncitial knots start appearing in the later stages of pregnancy and their number increases until term, at which time, knots are normally present on 11 to $30 \%$ of villi. Formation of knots on more than a third of villi is considered excessive. They are most frequent at the periphery of the cotyledons. In preeclampsia, the excessive formation of syncitial knots in the placenta is a consequence of obliterative changes in the fetal stem arteries [6]. The syncitiotrophoblastic knotting is an indicator of chorionic villous capillary proliferation or exaggerated senescence induced by hypoxia due to fetal or maternal pathologic conditions $[10,11]$. In the present study 4 out of $6(66.6 \%)$ cases of mild, 9 cases $(56.2 \%)$ of moderate, 23 cases $(82.1 \%)$ of severe pregnancy induced hypertension showed increased prominent syncitial knots. Syncitial knots are increased with increasing maternal under perfusion [2]and toxemia [6-8,13].

D) Stromal fibrosis: Stromal fibrosis is a feature of stem villous maturation and begins centrally in large stem villi about 15 weeks gestation. It progressively replaces the stroma of stem and immature intermediate villi with collagen. Generally, $<3 \%$ of term villi show fibrosis. The available evidence indicates that villous fibrosis may be a marker of reduced placental perfusion and associated with fetal growth restriction [11]. The two factors responsible for stromal fibrosis are normal aging and a reduced utero-placental blood flow [7].

The villi distal to an occluded vessel show a sequence of distinctive alterations. Early changes include karyorrhexis of intravascular, endothelial, and villous stromal cells with destruction of capillaries and extravasation of red cells. The stroma is mineralized and may be hypercellular. This constellation of alterations, currently termed villous stromal vascular karyorrhexis, is identical to hemorrhagic endovasculitis as originally described by Sander. Eventually, the villi become fibrotic with bland, densely collagenized, hyalinized stroma (fibrotic avascular villi). The surrounding syncytiotrophoblast is preserved and often knotted. Larger stem vessels distal to the occlusion show fibromuscular sclerosis. The thrombosed vessel resulting in these distal villous alterations may or may not be apparent depending on the plane of sectioning [2] Stromal fibrosis was seen in 1,9 and 18 cases- 16.6, 56.2 and $64.3 \%$ of mild, moderate and severe PIH cases in the present study. Narsimha and Ananya have shown increasing severity of stromal fibrosis with increasing toxemia [6,7]. Incidence of fibrosis of villi was also increased in hypertensive patients compared to normal according to Bar et al [9].

E) Cytotrophoblastic proliferation: Increase in the number of cytotrophoblasts reflects proliferation of precursor cells beyond the need of growth, survival and functional integrity of the syncitiotrophoblastic layer. Hypoxia enhances apoptosis in the syncytium, damages the syncitiotrophoblast and hinders differentiation which also stimulates the cytotrophoblastic cells by triggering mitotic rate, shortening the cell cycle and changes in the molecular and functional phenotype of the trophoblasts. Increased cytotrophoblastic proliferations was observed to be positively correlating with increasing hypertension $(\mathrm{p}<0.05)$ in the present study- $16.6 \%, 25 \%$ and $64.3 \%$ of mild, moderate and severe PIH cases. Similar trend has been noted in other studies [68]. Kartheek et al recorded abnormal cytotrophoblastic proliferation in $36.36 \%$ of hypertensive pregnancies as compared to normal ones [14].

F) Intervillous haemorrhage: Is of fetal origin but can rarely be due to trauma to the wall of the spiral artery. It is often associated with an infarct in the periphery of the same cotyledon [11]. Intervillous hematomas are clots in the intervillous space. They are found in $36-48 \%$ of placentas. Intervillous hematomas are thought to be initiated by fetal bleeding into the intervillous space through ruptured vasculosyncitial membranes. Small amounts of fetal blood can be demonstrated, although most of the clot is composed of maternal blood (Figure 4).

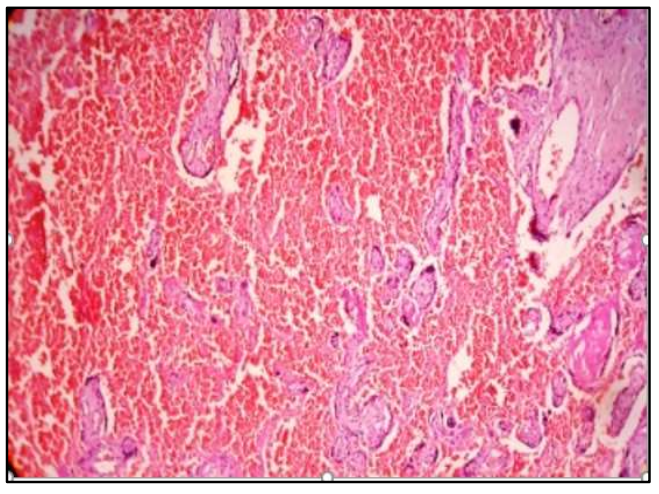

Fig-4: Intervillous haemorrhage (H\&E 10X). 
Factors cited as potential causes of villous damage resulting in fetomaternal hemorrhage include trauma, amniocentesis, and external version. Basilar intervillous hematomas may have a different etiology. Intervillous hematomas are round or oval blood clots that may occur anywhere in the intervillous space but are most common midway between the chorionic and basal plates. They begin as red, fluid, or semifluid blood and become progressively laminated and depigmented with age. They may be single, although multiple lesions are common. Most are 1-3 cm in diameter. Microscopically, the thrombi consist of layered red cells and fibrin, the proportion of fibrin increasing as the lesion ages. Villi displaced to the margins of the clot may be infarcted or fibrotic and avascular [2]. In the current study, $50 \%$ cases of moderate and $42.8 \%$ of severe pregnancy induced hypertension showed intervillous hemorrhage. Identical figures of $44.4 \%$ and $25 \%-35 \%$ have been demonstrated by others $[7,10]$.

G) Calcification (34\%): Common in areas of degenerating trophoblasts, it is dystrophic in nature. Although it occurs mainly in the decidual plate, it can occur any were in the placental tissue. Goswami et al recorded significant placental calcification in cases of PIH. The calcium deposits were mostly observed in the villi and basement membrane of the villi, strongly suggestive of uteroplacental insufficiency because of narrow lumen [4]. Calcification is not characteristic of any particular disorder [11].

In the present study calcification was seen in $31.2 \%$ and $42.8 \%$ of moderate and severe PIH cases. The incidence of calcification in other studies were $26.9 \%$ and $60.5 \%$ (7,11); Maimoona et al have shown an incidence of $15.38 \%$, through $30 \%$ to $50 \%$ in mild, moderate and severe PIH [8]. According to a study by Karun Jain, calcification was seen in $20 \%$ of PIH cases without IUGR and $41.37 \%$ in PIH cases with IUGR [10]. In the current study, there was muscular hypertrophy of the arterial wall in the basal plate (Figure 5). The muscular walls of the affected vessels in the decidua parietalis were thickened, and there was marked luminal narrowing. Mural hypertrophy is diagnosed when the mean wall diameter is greater than $30 \%$ of the overall vessel diameter.

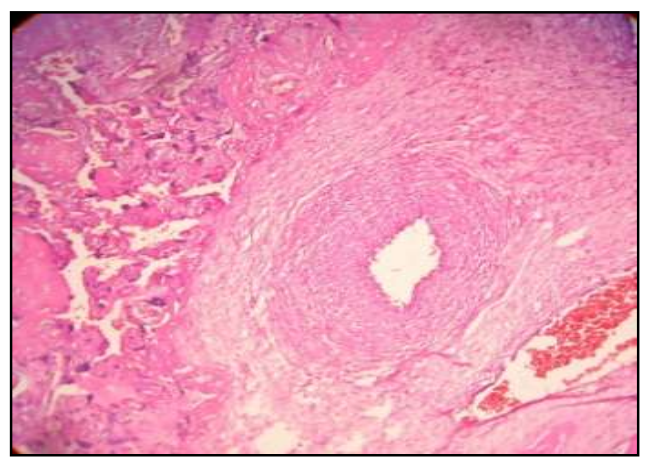

Fig-5: Mural hypertrophy of basal artery (H\&E 10X).

The present study had a case of severe PIH where the umbilical cord showed remnants of vitellointestinal duct (Figure 6). Traces of the omphalomesenteric duct, which connects the fetal ileum and the yolk sac in the early embryo, are infrequent, occurring in $1.5 \%$ of umbilical cords. These remnants are usually discontinuous, located peripherally, and lined by columnar cells resembling intestinal epithelium. The present study also observed squamous metaplasia of the membranes in some isolated cases (Figure 7). Foci of squamous metaplasia are slightly elevated, sometimes targetoid, pearly-white macules that tend to be most numerous at the site of cord insertion; foci of squamous epithelium, with or without keratinization, have a sharp transition with the surrounding amnionic epithelium. Squamousmetaplasia has no clinical significance, but it is important to distinguish from amnion nodosum, which it superficially resembles grossly [2].

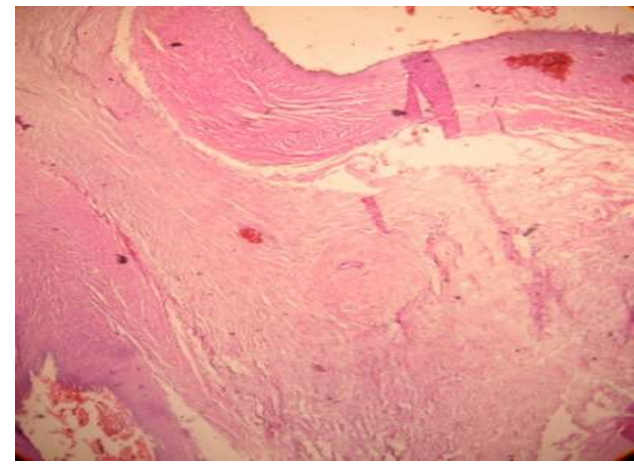

Fig-6: Vitellointestinal duct in umbilical cord (H\&E, 40X). 


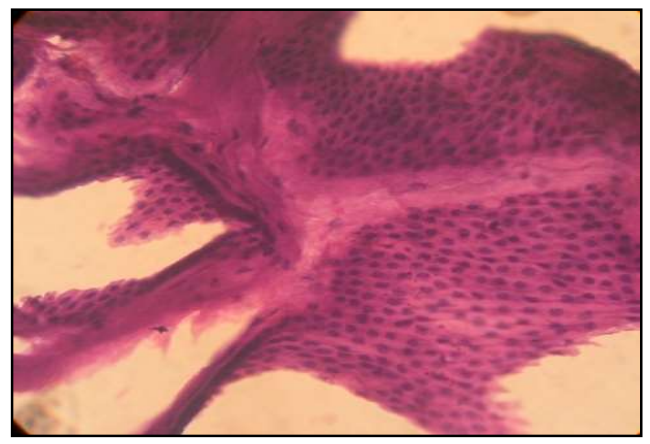

Fig-7: Squamous metaplasia of membranes (H\&E, 40X).

\section{Limitations of the present study}

1.The correlation between placental lesions and severity of maternal hypertension was poor, probably because the sample size was small. A greater sample size gives a definite association of the lesions with hypertension.

2. The co-morbid conditions associated with PIH were not taken into consideration. The contribution of these conditions to placental histology needs to be determined.

\section{Conclusion}

The severity of the pathological changes in increased with the degree of PIH. A statistically significant association between the microscopy and degree of PIH was seen in fibrinoid necrosis and cytotrophoblastic maturation. Correlation between placental lesions and severity of maternal hypertension is poor. There is an overlap of these changes in different disorders of pregnancy. The origin of the placental changes in PIH is yet to be established. The morphology of the changes showed that there are findings apart from that due to maternal under perfusion. These findings are to be studied in association with the pathophysiologic processes to draw conclusions on the pathogenesis.

\section{What this study adds to existing knowledge?}

1.The frequency of histological changes increased with the increasing severity of PIH. Hence early detection and management of hypertension reduces foetal and maternal morbidity.

2. As the hypertension increased, the changes associated with maternal and placental ischemia increased significantly; thus establishing the fact that under perfusion of maternal and fetal circulation causes hypertension and its consequent complications.

\section{Author's contribution}

Dr. Rajyalakshmi and Dr. Vani have contributed to conception and design of the study and to compilation and interpretation of data. Dr. Jijiya Bai have given final approval of the drafted article.

Acknowledgements: The authors wish to acknowledge Dr B.R. Yelikar, MD for reviewing the manuscript and helping in preparing the final copy. Authors are also thankful to Dr
Md. Sikinder Hayath, MD, DCP for his guidance in the study.

Funding: No funding sources

Conflict of interest: None declared

Ethical Approval: This study was approved by the Institutional Ethics Committee

\section{References}

1. Ogge G, Chaiworapongsa T, Romero R, Hussein Y, Kusanovic JP, Yeo L, et al. Placental lesions associated with maternal underperfusion are more frequent in earlyonset than in late-onset preeclampsia. J Perinat Med. 2011;39(6):641-652. doi: 10.1515/JPM.2011.098

2. Gersell DJ, Kraus FT. Diseases of the Placenta. In: Kurman RJ, Ellenson LH, Ronnett BM (Eds). Blaustein's Pathology of the Female Genital Tract. $6^{\text {th }}$ ed. Springer 2011.

3. Ezeigwe CO, Okafor CI, Eleje GU, Udigwe GO, Anyiam DC. Placental peripartum pathologies in women with preeclampsia and eclampsia. Obstet Gynecol Int. 2018; 2018:9462938. doi: 10.1155/2018/9462938. eCollection 2018.

4. Goswami P, Lata H, Memon S, Khaskhelli LB. Excessive placental calcification observed in PIH patients and its relation to fetal outcome. JLUMHS. 2012;11(03):143-148.

5. Fox H. The histopathology of placental insufficiency. J Clin Pathol. 1976;29:10:1-8.

6. Agrawal A, Kumar K, Kumar S, Nayak S, Khushboo, Yadav N. Spectrum of placental changes in Toxemia of 
pregnancy: A case series study. Int $\mathrm{J}$ Clinic Obstet Gynaecol. 2017;1(1):8-13.

7. Narasimha A, Vasudeva DS. Spectrum of changes in placenta in toxemia of pregnancy. Indian $\mathrm{J}$ Pathol Microbiol. 2011;54(1):15-20. doi:10.4103/0377-4929. 77317.

8. Ahmed M, Daver RG. Study of placental changes in pregnancy induced hypertension. Int $\mathrm{J}$ Reprod Contracept Obstet Gyneco.1 2013;2(4):524-527. doi: 10.5455/23201770.ijrcog20131207.

9. Bar PK, Ghosh S, Gayen P,Mandal S, De A, Biswas A. Morphologixcal study of placenta in hypertensive disorders in pregnancy. Trop J Path Micro. 2019;5(6):366-373. doi: 10.17511/jopm. 2019.i6.06.

10. Jain K, Kavi V, Raghuveer CV, Sinha R. Placental pathology in pregnancy-induced hypertension (PIH) with or without intrauterine growth retardation. Indian J Pathol Microbiol. 2007;50(3):533-537.
11. Mohan SR, Jyosna, Anil S, ChandraSekar S. Morphological changes in placentas of normal and high risk pregnancies-2 years study in MGM hospital. IAIM 2017;4(5):61-78.

12. Christopher P. Crum. The Female Genital Tract. In: Kumar, Abbas and Fausto (Eds). Robbins and Cotran Pathologic Basis of Disease. $7^{\text {th }}$ Ed. Elsevier 2007.

13. Salmani D, Purushothaman S, Somashekara SC, Gnanagurudasan E, Sumangaladevi K, Harikishan R et al. Study of structural changes in placenta in pregnancyinduced hypertension. J Nat Sci Biol Med. 2014;5(2): 352355. doi: 10.4103/0976-9668.136182.

14. Kartheek BVS, Atla B, Prasad U, Namballa U, Prabhakula S. A study of placental morphology and correlation with colour doppler ultrasonography, maternal and neonatal outcome in high risk pregnancies. Int $\mathrm{J}$ Res Med Sci. 2018;6(10):3364-3369. doi: http://dx.doi.org/ 10.18203/ 2320-6012.ijrms20184047.

\section{How to cite this article?}

S. Rajyalakshmi, Vani B S, Jijiya Bai P. Placental pathology in different degrees of pregnancy induced hypertension (PIH). Trop J Path Micro 2019;5(12): 988-996.doi:10.17511/ jopm.2019.i12.04 